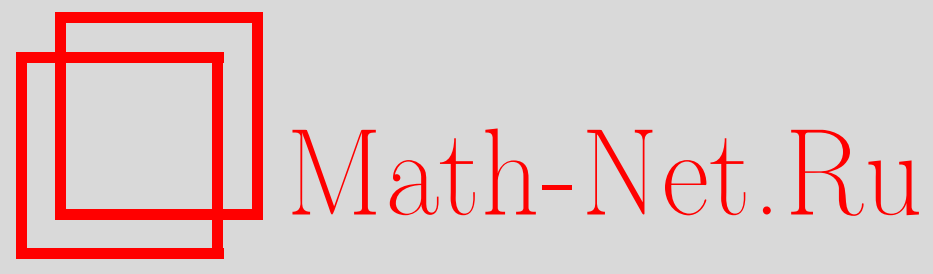

С. А. Бочкарев, С. В. Лекомцев, Исследование влияния граничных условий на устойчивость коаксиальных цилиндрических оболочек, взаимодействующих с текущей жидкостью, Вестн. Сам. гос. техн. ун-та. Сер. Физ.-мат. науки, 2012, выпуск 3(), 88-101

DOI: https://doi.org/10.14498/vsgtu1051

Использование Общероссийского математического портала Math-Net.Ru подразумевает, что вы прочитали и согласны с пользовательским соглашением

http: //www . mathnet.ru/rus/agreement

Параметры загрузки:

IP: 3.91 .87 .62

26 апреля 2023 г., 18:32:26

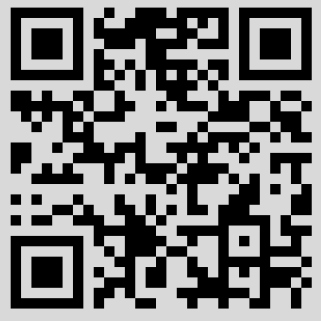


УДК 533.6.013.42

\title{
ИССЛЕДОВАНИЕ ВЛИЯНИЯ ГРАНИЧНЫХ УСЛОВИЙ НА УСТОЙЧИВОСТЬ КОАКСИАЛЬНЫХ ЦИЛИНДРИЧЕСКИХ ОБОЛОЧЕК, ВЗАИМОДЕЙСТВУЮЩИХ С ТЕКУЩЕЙ ЖИДКОСТЬЮ
}

\author{
С. А. Бочкарёв, С.В. Лекомцев \\ Институт механики сплошных сред УрО РАН, \\ 614013, Россия, Пермь, ул. Акад. Королёва, 1. \\ E-mails: bochkarev@icmm.ru, lekomtsev@icmm.ru
}

\begin{abstract}
Исследовано динамическое поведение упругих коаксиальных иилиндрических оболочек, взаимодействующих с идеалъной сжимаемой жидкостъю. Для оболочек используется классическая теория оболочек и приниип возможных перемешений в качестве математической формулировки. Поведение жидкости описъвается в рамках потенциальной теории. В результате задача сводится к совместному решению четырёх систем уравнений, осуществляемому методом конечных элементов. Для оболочек с различными комбинациями граничных условий выполнено исследование влияния величины кольцевого зазора на границу гидроупругой устойчивости.
\end{abstract}

Ключевые слова: коаксиальные оболочки, потенииальная жидкость, метод конечных элементов, устойчивость, дивергениия, флаттер.

Введение. Цилиндрические конструкции различных конфигураций широко используются в нефтехимическом производстве и атомной промышленности для хранения и переноса жидкостей под высокими давлением и температурой. В частности, система трубопроводов может быть смоделирована или как одиночная круговая цилиндрическая оболочка, или как система коаксиальных оболочек, содержащих внутренний осевой поток жидкости. Коаксиальные оболочки, которые кроме этого могут взаимодействовать с потоком жидкости, расположенным в канале между двумя оболочками или обоими потоками, являются также структурными элементами многих технических приложений. Несмотря на это, количество работ, посвященных исследованию их динамического поведения, является незначительным.

Динамика и устойчивость коаксиальных цилиндрических оболочек, содержащих невязкую потенциальную жидкость, исследовалась для жёстко закреплённых с двух торцов [1] и консольно закреплённых оболочек [2]. В [1] установлено, что в случае кольцевого течения жидкости потеря устойчивости в виде дивергенции наблюдается при существенно меньших скоростях жидкости, чем в случае внутреннего течения, а совместное течение двух потоков еще больше дестабилизирует систему. В [2] показано, что потеря устойчивости осуществляется в виде флаттера по одной форме колебаний, а совместное течение двух потоков может стабилизировать или дестабилизировать систему в зависимости от их скоростей.

При использовании моделей, учитывающих вязкость среды, могут приниматься во внимание как стационарные [2-5], так и нестационарные [5] си-

Сергей Аркадъевич Бочкарёв (к.ф.-м.н.), научный сотрудник, отдел комплексных проблем механики деформируемых твёрдых тел. Сергей Владимирович Лекомиев, аспирант, отд. комплексных проблем механики деформируемых твёрдых тел. 
лы вязкого сопротивления. Для получения стационарных сил используется усреднённые по времени уравнения Навье-Стокса для развитого турбулентного несжимаемого потока жидкости. Показано [2, 3], что они оказывают существенное влияние на критические скорости течения жидкости. Нестационарные силы вязкого сопротивления определяются из линеаризованных уравнений Навье-Стокса с использованием численной процедуры, основанной на конечно-разностном методе. В [5] продемонстрировано, что эта модель лучше согласуется с экспериментальными данными [8,9], чем модель, учитывающая только стационарные вязкостные эффекты. В работах [6,7] в рамках численного решения задачи для невязкой и вязкой жидкости обнаружено существенное расхождение с представленными в $[1,2]$ данными для тех случаев, когда потеря устойчивости коаксиальных оболочек осуществляется на высоких модах колебаний.

В перечисленных работах рассматриваются варианты, когда обе оболочки имеют одинаковые граничные условия. В настоящей статье в рамках разработанного конечно-элементного решения задачи выполнено исследование влияния различных комбинаций граничных условий, задаваемых на торцах каждой из оболочек. Оценен вид потери устойчивости, осуществляющийся при различной величине кольцевого зазора между внутренней и внешней оболочками, разных видах течения жидкости, включая совместное течение внутреннего и кольцевого потоков с одинаковой скоростью.

1. Основные соотношения. Рассматривается коаксиальная система, состоящая из двух упругих цилиндрических оболочек длиной $L$ : внутренняя радиусом $a$ и толщиной $h_{i}$ и внешняя радиусом $b$ и толщиной $h_{o}$. Эта система коаксиальных оболочек взаимодействует с двумя потоками идеальной сжимаемой жидкости. Один поток, содержащий жидкость с удельной плотностью $\rho_{f}^{i}$ и скоростью звука в нём $c_{i}$, течёт во внутренней оболочке со скоростью $U_{i}$, а другой $\left(\rho_{f}^{o}, c_{o}, U_{o}\right)$ - в кольцевом канале между двумя оболочками. Решение задачи заключается в определении такой скорости внутреннего или кольцевого потоков жидкости или их комбинации, при которой система коаксиальных оболочек теряет устойчивость.

Потенциальное движение идеальной сжимаемой жидкости описывается волновым уравнением, которое в цилиндрических координатах $(r, \theta, x)$ для внутреннего течения, занимающего объём $V_{f}^{i}$, записывается в виде [10]

$$
\nabla^{2} \phi_{i}=\frac{\partial^{2} \phi_{i}}{\partial r^{2}}+\frac{1}{r^{2}} \frac{\partial^{2} \phi_{i}}{\partial \theta^{2}}+\frac{\partial^{2} \phi_{i}}{\partial x^{2}}+\frac{1}{r} \frac{\partial \phi_{i}}{\partial r}=\frac{1}{c_{i}^{2}}\left[\frac{\partial}{\partial t}+U_{i} \frac{\partial}{\partial x}\right]^{2} \phi_{i}
$$

где $\phi_{i}$ - потенциал возмущения скорости. Аналогичное уравнение (с заменой индекса $i$ на $о$ ) записывается для кольцевого течения, занимающего объём $V_{f}^{o}$ и описываемого потенциалом $\phi_{o}$.

Для вычисления давления $p_{i}$, действующего со стороны жидкости на упругую поверхность внутренней оболочки $\left(S_{\sigma}^{i}=S_{f}^{i} \cap S_{s}^{i}\right)$, используется уравнение Бернулли

$$
p_{i}=-\rho_{f}^{i}\left(\frac{\partial \phi_{i}}{\partial t}+U_{i} \frac{\partial \phi_{i}}{\partial s_{i}}\right) .
$$

Здесь $s_{i}$ - меридиональная координата внутренней оболочки, а $S_{f}^{i}, S_{s}^{i}$ - поверхности, ограничивающие объёмы внутреннего потока жидкости и внут- 
ренней оболочки. Аналогичное уравнение (с заменой индекса $i$ на o) записывается для давления кольцевого потока $p_{o}$ на поверхности раздела внешней оболочки и кольцевого потока $S_{\sigma}^{o}=S_{f}^{o} \cap S_{s}^{o}$. На поверхности раздела «внутренняя оболочка - внутренний поток» жидкости $S_{\sigma}^{i}$ задается условие непроницаемости

$$
\frac{\partial \phi_{i}}{\partial n}=\frac{\partial w_{i}}{\partial t}+U_{i} \frac{\partial w_{i}}{\partial s_{i}}
$$

где $n$ - нормаль к поверхности $S_{\sigma}^{i}, w_{i}$ - нормальная составляющая вектора перемещений внутренней оболочки. Для кольцевого течения задаются следующие условия:

$$
\begin{gathered}
\frac{\partial \phi_{o}}{\partial n}=\frac{\partial w_{i}}{\partial t}+U_{o} \frac{\partial w_{i}}{\partial s_{i}} \quad \text { на } \quad S_{f}^{o} \cap S_{s}^{i}, \\
\frac{\partial \phi_{o}}{\partial n}=\frac{\partial w_{o}}{\partial t}+U_{o} \frac{\partial w_{o}}{\partial s_{o}} \quad \text { на } \quad S_{\sigma}^{o} .
\end{gathered}
$$

Потенциалы возмущения скорости на входе в оболочки и выходе из них подчиняются следующим граничным условиям:

$$
x=0: \phi_{i}=\phi_{o}=0, \quad x=L: \frac{\partial \phi_{i}}{\partial x}=\frac{\partial \phi_{o}}{\partial x}=0 .
$$

Уравнение для потенциала возмущения скорости (1) вместе с граничными условиями (3) и (6) сводится к системе уравнений с помощью метода Бубнова-Галёркина. Для внутреннего потока соотношения примут следующий вид [11]:

$$
\begin{aligned}
\sum_{l=1}^{m_{f}^{i}}\left[\int_{V_{f}^{i}}\left(\frac{\partial F_{l}^{i}}{\partial r} \frac{\partial F_{k}^{i}}{\partial r}+\frac{1}{r^{2}} \frac{\partial F_{l}^{i}}{\partial \theta} \frac{\partial F_{k}^{i}}{\partial \theta}+\left(1-M_{i}^{2}\right) \frac{\partial F_{l}^{i}}{\partial x} \frac{\partial F_{k}^{i}}{\partial x}\right) d V\right] \phi_{a l}^{i}+ \\
\quad+\sum_{l=1}^{m_{f}^{i}}\left[\int_{V_{f}^{i}} \frac{2 U_{i}}{c_{i}^{2}} \frac{\partial F_{l}^{i}}{\partial x} F_{k}^{i} d V \dot{\phi}_{a l}^{i}+\int_{V_{f}^{i}} \frac{F_{l}^{i} F_{k}^{i}}{c_{i}^{2}} d V \ddot{\phi}_{a l}^{i}\right]- \\
-\sum_{p=1}^{m_{s}^{i}}\left[\int_{S_{\sigma}^{i}} \bar{N}_{p}^{i} F_{k}^{i} d S \dot{w}_{a p}^{i}+\int_{S_{\sigma}^{i}} U_{i} \frac{\partial \bar{N}_{p}^{i}}{\partial s} F_{k}^{i} d S w_{a p}^{i}\right]=0, \quad k=1,2, \ldots m_{f}^{i} .
\end{aligned}
$$

Здесь $m_{f}^{i}$ и $m_{s}^{i}$ - число конечных элементов, на которые разбиваются области жидкости $V_{f}^{i}$ и внутренней оболочки $V_{s}^{i} ; \phi_{a l}^{i}$ и $w_{a p}^{i}$-узловые значения потенциала возмущения скорости жидкости и перемещений оболочки; $M_{i}=$ $=U_{i} / c_{i}$ - число Маха внутреннего потока; $F_{i}$ и $\bar{N}_{i}-$ функции формы для потенциала возмущения скорости внутреннего потока и нормальной составляющей вектора перемещения внутренней оболочки. Аналогичное уравнение (с заменой индекса $i$ на $o$ ) записывается для кольцевого течения, в котором также будет присутствовать дополнительные слагаемые в результате учёта условия (4). 
Оболочки рассматриваются в рамках гипотез Кирхгофа-Лява, согласно которым компоненты вектора деформаций срединной поверхности, изменения кривизн и кручения в координатной системе $(s, \theta, z)$ записываются следующим образом [12]:

$$
\begin{aligned}
\varepsilon_{1}^{i}=\frac{\partial u_{i}}{\partial s}, & \varepsilon_{2}^{i}=\frac{1}{a}\left(\frac{\partial v_{i}}{\partial \theta}+w_{i}\right), & \varepsilon_{12}^{i}=\frac{1}{a} \frac{\partial u_{i}}{\partial \theta}+\frac{\partial v_{i}}{\partial s}, \\
\kappa_{1}^{i}=-\frac{\partial^{2} w_{i}}{\partial s^{2}}, & \kappa_{2}^{i}=\frac{1}{a^{2}}\left(\frac{\partial v_{i}}{\partial \theta}-\frac{\partial^{2} w_{i}}{\partial \theta^{2}}\right), & \kappa_{12}^{i}=\frac{1}{a}\left(\frac{\partial v_{i}}{\partial s}-\frac{\partial^{2} w_{i}}{\partial s \partial \theta}\right),
\end{aligned}
$$

где $u_{i}$ и $v_{i}$ - меридиональная и окружная составляющие вектора перемещений внутренней оболочки.

Физические соотношения, устанавливающие связь между вектором обобщённых усилий и моментов $\boldsymbol{T}_{i}$ и вектором обобщённых деформаций $\boldsymbol{\varepsilon}_{i}=$ $=\left\{\varepsilon_{1}^{i}, \varepsilon_{2}^{i}, \varepsilon_{12}^{i}, \kappa_{1}^{i}, \kappa_{2}^{i}, 2 \kappa_{12}^{i}\right\}^{\top}$, представляются в матричном виде

$$
\boldsymbol{T}_{i}=\left\{T_{11}^{i}, T_{22}^{i}, T_{12}^{i}, M_{11}^{i}, M_{22}^{i}, M_{12}^{i}\right\}^{\top}=\mathbf{D}_{i} \varepsilon_{i} .
$$

Для изотропного материала ненулевые компоненты матрицы жесткостей $\mathbf{D}_{i}$ определяются известным образом через модуль упругости $E_{i}$, коэффициент Пуассона $\nu_{i}$ и модуль сдвига.

Для математической формулировки задачи используется принцип возможных перемещений с учётом работы сил инерции, который для внутренней оболочки в матричной форме может быть записан следующим образом:

$$
\int_{S_{i}} \delta \boldsymbol{\varepsilon}_{i}^{\top} \boldsymbol{T}_{i} d S+\int_{S_{i}} \delta \boldsymbol{d}_{i}^{\top} \rho_{0}^{i} \ddot{\boldsymbol{d}}_{i} d S-\int_{S_{i}} \delta \boldsymbol{d}_{i}^{\top} \boldsymbol{P}_{i} d S=0 .
$$

Здесь $\boldsymbol{d}_{i}$ и $\boldsymbol{P}_{i}=\left.p_{i}\right|_{r=a}-\left.p_{o}\right|_{r=a}-$ векторы обобщенных перемещений и поверхностных нагрузок, $\rho_{0}^{i}=\int_{h_{i}} \rho_{s}^{i} d z, \rho_{s}^{i}$ - удельная плотность материала внутренней оболочки. Уравнения, аналогичные (7)-(8), записываются и для внешней оболочки (при замене индекса $i$ на $о$ и $a$ на $b$ ), причём $\boldsymbol{P}_{o}=\left.p_{o}\right|_{r=b}$.

2. Численная реализация. Для численной реализации используется полуаналитический вариант метода конечных элементов, основанный на представлении решения в виде ряда Фурье по окружной координате $\theta$ :

$$
u=\sum_{j=0}^{\infty} u_{j} \cos j \theta, v=\sum_{j=0}^{\infty} v_{j} \sin j \theta, w=\sum_{j=0}^{\infty} w_{j} \cos j \theta, \phi_{a}=\sum_{j=0}^{\infty} \phi_{j} \cos j \theta .
$$

Здесь $j$ - номер гармоники. В результате исходная двумерная задача сводится к совокупности одномерных задач для каждой из гармоник ряда Фурье. Компоненты вектора перемещений и потенциал возмущения скорости внутри элемента определяются через узловые неизвестные (например, для внутренней оболочки и внутреннего потока)

$$
\begin{gathered}
\boldsymbol{U}_{i}=\left\{u_{i}, v_{i}, w_{i}\right\}^{\mathrm{T}}=\mathbf{N}_{i} \boldsymbol{\delta}_{i}^{e}, \\
\boldsymbol{\phi}_{a}^{i}=\mathbf{F}_{i} f_{i}^{e},
\end{gathered}
$$


где $\boldsymbol{\delta}_{i}^{e}$ и $f_{i}^{e}$ - вектора узловых неизвестных, $\mathbf{N}_{i}$ и $\mathbf{F}_{i}$ - матрицы функций формы, которые формируются в зависимости от типа выбранного конечного элемента. Для жидкости используется треугольный элемент с линейной аппроксимацией. Для оболочек применяется двухузловой конечный элемент в форме усеченного конуса с аппроксимацией меридиональной и окружной компонент вектора перемещений линейным полиномом, а нормальной компоненты - кубическим полиномом.

С учётом (9) связь вектора деформаций внутренней оболочки $\varepsilon_{i}$ с вектором узловых неизвестных оболочечного конечного элемента $\boldsymbol{\delta}_{i}^{e}$ представляется в виде

$$
\varepsilon_{i}=\mathbf{B}_{i} \boldsymbol{\delta}_{i}^{e}
$$

Подставляя в уравнение (8) значение для давления в форме (2) и используя стандартные процедуры метода конечных элементов с учётом (9)-(11), получим матричное соотношение

$$
\mathbf{K}_{i}^{s} \boldsymbol{d}_{i}+\mathbf{M}_{i}^{s} \ddot{\boldsymbol{d}}_{i}+\mathbf{C}_{i}^{s} \dot{\boldsymbol{f}}_{i}+\mathbf{A}_{i}^{s} \boldsymbol{f}_{i}=0 .
$$

Здесь $\boldsymbol{f}_{i}$ - вектор обобщенных узловых значений потенциала возмущения скорости внутреннего потока и

$$
\begin{gathered}
\mathbf{K}_{s}^{i}=\sum_{m_{s}^{i}} \int_{S_{s}^{i}} \mathbf{B}_{i}^{\top} \mathbf{D}_{i} \mathbf{B}_{i} d S, \quad \mathbf{M}_{s}^{i}=\sum_{m_{s}^{i}} \int_{S_{s}^{i}} \mathbf{N}_{i}^{\top} \rho_{0}^{i} \mathbf{N}_{i} d S, \\
\mathbf{A}_{s}^{i}=\sum_{m_{s}^{i}} \int_{S_{\sigma}^{i}} \rho_{f}^{i} U_{i} \overline{\mathbf{N}}_{i}^{\top} \frac{\partial \mathbf{F}_{i}}{\partial s} d S, \quad \mathbf{C}_{s}^{i}=\sum_{m_{s}^{i}} \int_{S_{\sigma}^{i}} \rho_{f}^{i} \overline{\mathbf{N}}_{i}^{\top} \mathbf{F}_{i} d S .
\end{gathered}
$$

Уравнение (5) в матричном виде с учётом представлений (9), (10) принимает следующий вид:

$$
\mathbf{K}_{f}^{i} \boldsymbol{f}_{i}+\mathbf{M}_{f}^{i} \ddot{\boldsymbol{f}}_{i}+\mathbf{C}_{f}^{c i} \dot{\boldsymbol{f}}_{i}+\mathbf{A}_{f}^{c i} \boldsymbol{f}_{i}+\mathbf{C}_{f}^{i} \dot{\boldsymbol{d}}_{i}+\mathbf{A}_{f}^{i} \boldsymbol{d}_{i}=0
$$

где

$$
\begin{gathered}
\mathbf{K}_{f}^{i}=\sum_{m_{f}^{i}} \int_{V_{f}^{i}}\left(\frac{\partial \mathbf{F}_{i}^{\top}}{\partial r} \frac{\partial \mathbf{F}_{i}}{\partial r}+\frac{1}{r^{2}} \frac{\partial \mathbf{F}_{i}^{\top}}{\partial \theta} \frac{\partial \mathbf{F}_{i}}{\partial \theta}+\frac{\partial \mathbf{F}_{i}^{\top}}{\partial x} \frac{\partial \mathbf{F}_{i}}{\partial x}\right) d V, \mathbf{M}_{f}^{i}=\sum_{m_{f}^{i}} \int_{V_{f}^{i}} \frac{\mathbf{F}_{i}^{\top} \mathbf{F}_{i}}{c_{i}^{2}} d V, \\
\mathbf{A}_{f}^{i}=-\sum_{m_{s}^{i}} \int_{S_{\sigma}^{i}} U_{i} \mathbf{F}_{i}^{\top} \frac{\partial \overline{\mathbf{N}}_{i}}{\partial s} d S, \mathbf{A}_{f}^{c i}=-\sum_{m_{f}^{i}} \int_{V_{f}^{i}} M_{i}^{2} \frac{\partial \mathbf{F}_{i}^{\top}}{\partial s} \frac{\partial \mathbf{F}_{i}}{\partial s} d V \\
\mathbf{C}_{f}^{i}=-\sum_{m_{s}^{i}} \int_{S_{\sigma}^{i}} \mathbf{F}_{i}^{\top} \overline{\mathbf{N}}_{i} d S, \mathbf{C}_{f}^{c i}=\sum_{m_{f}^{i}} \int_{V_{f}^{i}} \frac{2 U_{i}}{c_{i}^{2}} \frac{\partial \mathbf{F}_{i}^{\top}}{\partial x} \mathbf{F}_{i} d V .
\end{gathered}
$$

Таким образом, анализ устойчивости коаксиальных оболочек, содержащих текущую жидкость, сводится к совместному решению четырёх систем уравнений (12) и (13). Объединённая система уравнений может быть записана следующим образом:

$$
(\mathbf{K}+\mathbf{A})\left\{\boldsymbol{d}_{i}, \boldsymbol{f}_{i}, \boldsymbol{d}_{o}, \boldsymbol{f}_{o}\right\}^{\top}+\mathbf{M}\left\{\ddot{\boldsymbol{d}}_{i}, \ddot{\boldsymbol{f}}_{i}, \ddot{\boldsymbol{d}}_{o}, \ddot{\boldsymbol{f}}_{o}\right\}^{\top}+\mathbf{C}\left\{\dot{\boldsymbol{d}}_{i}, \dot{\boldsymbol{f}}_{i}, \dot{\boldsymbol{d}}_{o}, \dot{\boldsymbol{f}}_{o}\right\}^{\top}=0
$$


где

$$
\begin{array}{ccc}
\mathbf{K} & =\operatorname{diag}\left\{\mathbf{K}_{\mathrm{s}}^{\mathrm{i}}, \mathbf{K}_{\mathrm{f}}^{\mathrm{i}}, \mathbf{K}_{\mathrm{s}}^{\mathrm{o}}, \mathbf{K}_{\mathrm{f}}^{\mathrm{o}}\right\}, & \mathbf{M}=\operatorname{diag}\left\{\mathbf{M}_{\mathrm{s}}^{\mathrm{i}}, \mathbf{M}_{\mathrm{f}}^{\mathrm{i}}, \mathbf{M}_{\mathrm{s}}^{\mathrm{o}}, \mathbf{M}_{\mathrm{f}}^{\mathrm{o}}\right\}, \\
\mathbf{A}=\left[\begin{array}{cccc}
0 & \mathbf{A}_{s}^{i} & 0 & \mathbf{A}_{s}^{o} \\
\mathbf{A}_{f}^{i} & \mathbf{A}_{f}^{c i} & 0 & 0 \\
0 & 0 & 0 & \mathbf{A}_{s}^{o} \\
\mathbf{A}_{f}^{o} & 0 & \mathbf{A}_{f}^{0} & \mathbf{A}_{f}^{c o}
\end{array}\right], & \mathbf{C}=\left[\begin{array}{cccc}
0 & \mathbf{C}_{s}^{i} & 0 & \mathbf{C}_{s}^{o} \\
\mathbf{C}_{f}^{i} & \mathbf{C}_{f}^{c i} & 0 & 0 \\
0 & 0 & 0 & \mathbf{C}_{s}^{o} \\
\mathbf{C}_{f}^{o} & 0 & \mathbf{C}_{f}^{o} & \mathbf{C}_{f}^{c o}
\end{array}\right] .
\end{array}
$$

Недостающие матрицы могут быть получены в результате замены индекса $i$ на 0 .

Представляя возмущенное движение оболочки и жидкости в виде

$$
\left(\boldsymbol{d}_{i}, \boldsymbol{f}_{i}, \boldsymbol{d}_{o}, \boldsymbol{f}_{o}\right)=\left(\boldsymbol{q}_{i}, \boldsymbol{g}_{i}, \boldsymbol{q}_{o}, \boldsymbol{g}_{o}\right) \exp \left(i^{*} \lambda t\right),
$$

где $\left(\boldsymbol{q}_{i}, \boldsymbol{g}_{i}, \boldsymbol{q}_{o}, \boldsymbol{g}_{o}\right)$ - некоторые функции координат, $i^{*}=\sqrt{-1}, \lambda=\lambda_{1}+i^{*} \lambda_{2}-$ характеристический показатель, окончательно получим

$$
\left(\mathbf{K}+\mathbf{A}-\lambda^{2} \mathbf{M}+i^{*} \lambda \mathbf{C}\right)\left\{\boldsymbol{q}_{i}, \boldsymbol{g}_{i}, \boldsymbol{q}_{o}, \boldsymbol{g}_{o}\right\}^{\top}=0 .
$$

Решение задачи сводится к вычислению и анализу собственных значений системы (14). Для вычисления комплексных собственных значений применяется алгоритм на основе метода Мюллера [13]. Несмотря на некоторые недостатки, возникающие при вычислении комплексных собственных значений, этот метод является достаточно эффективным, поскольку не требует сведения к стандартной или обобщенной проблеме на собственные значения. Для повышения вычислительной эффективности алгоритма используется перенумерация степеней свободы системы (14), основанная на обратном алгоритме Катхилла-Макки [14].

3. Результаты численных экспериментов. При взаимодействии коаксиальных оболочек с невязкой жидкостью различные граничные условия, задаваемые на торцах обеих оболочек, однозначно определяют вид потери устойчивости. Далее будут представлены результаты, демонстрирующие более сложное поведение, определяемое тем, что внутренняя и внешняя оболочки имеют разные комбинации граничных условий. Для них принимаются следующие обозначения: $C$ - жёсткое защемление $(u=v=w=\partial w / \partial s=0) ; S$ - свободно опертый край $(v=w=0) ; F$-свободный край. Например, комбинация $C C-S S$ означает, что внутренняя оболочка жёстко защемлена на обоих торцах, тогда как наружная оболочка свободно оперта.

Расчёты выполнялись при различной величине кольцевого зазора между внешней и наружной оболочками $k=(b-a) / a$. При этом неизменными оставались следующие характеристики: $L=1 \mathrm{M}, b=0,1 \mathrm{M}, h_{i}=h_{o}=5 \cdot 10^{-4} \mathrm{M}$, $\nu=0,3, E=2 \cdot 10^{11} \mathrm{H} / \mathrm{m}^{2}, \rho_{s}^{i}=\rho_{s}^{o}=7800 \mathrm{\kappa г} / \mathrm{M}^{3}, \rho_{f}^{i}=\rho_{f}^{o}=10^{3} \mathrm{\kappa г} / \mathrm{m}^{3}$. В представлении численных результатов используются безразмерные переменные $\Lambda_{i}=U_{i} \Delta_{i}, \Omega_{i}=\lambda a \Delta_{i}, \Omega_{i}^{1}=\operatorname{Re}\left(\Omega_{i}\right), \Omega_{i}^{2}=\operatorname{Im}\left(\Omega_{i}\right), \Delta_{i}=\left[\rho_{s}^{i}\left(1-\nu_{i}\right) / E_{i}\right]^{1 / 2}$ (для кольцевого течения $a$ заменяется на $b$ и $i$ на $o$ ).

С целью определения оптимальных параметров конечно-элементной сетки для систем с различными конфигурациями была проведена оценка сходимости решения. В результате выполненных численных экспериментов установлено, что приемлемую точность (наряду с комфортностью вычислений) 
обеспечивают сетки со следующим количеством конечных элементов: 40 для каждой из оболочек; 1600 - для жидкости (количество элементов для внутреннего и кольцевого течений определяется величиной зазора между оболочками).

Для тестирования разработанного численного алгоритма осуществлено сравнение с известными численно-аналитическими решениями, для оболочек с различными граничными условиями и жёсткой внешней оболочкой.

На рис. 1, а представлены результаты, полученные для коаксиальных оболочек, жёстко закреплённых с двух торцов. Здесь показана зависимость безразмерных действительных $\Omega_{i}^{1}$ (сплошные линии) и мнимых $\Omega_{i}^{2}$ (штрихпунктирные линии) частей трех первых собственных значений от безразмерной скорости внутреннего потока жидкости $\Lambda_{i}(m-$ число полуволн в меридиональном направлении, $\left.k=1 / 10, j=3, \Lambda_{o}=0\right)$. Потеря устойчивости при данных граничных условиях осуществляется в виде дивергенции - при увеличении скорости течения жидкости собственные значения уменьшаются до тех пор, пока действительная часть первой моды $(m=1)$ при скорости $\Lambda_{D}$ не станет равной нулю.

На рис. 1, б показаны результаты, полученные для консольно закреплённых оболочек. Здесь представлена диаграммы изменений трех первых безразмерных комплексных собственных значений $\Omega_{i}$ от различных значений безразмерной скорости внутреннего потока жидкости $\Lambda_{i}(k=1 / 10, j=2)$. Как и в случае одной оболочки с аналогичными граничными условиями, потеря устойчивости системы коаксиальных оболочек осуществляется в виде флаттера по одной форме колебаний - с увеличением скорости потока мнимая часть второй моды колебаний $(m=2)$ при скорости становится отрицательной.

На обоих рисунках маркерами обозначены результаты расчётов, полученных в [1] и [2]. Для рассмотренных конфигураций представленные данные демонстрируют хорошее совпадение полученных и известных результатов.

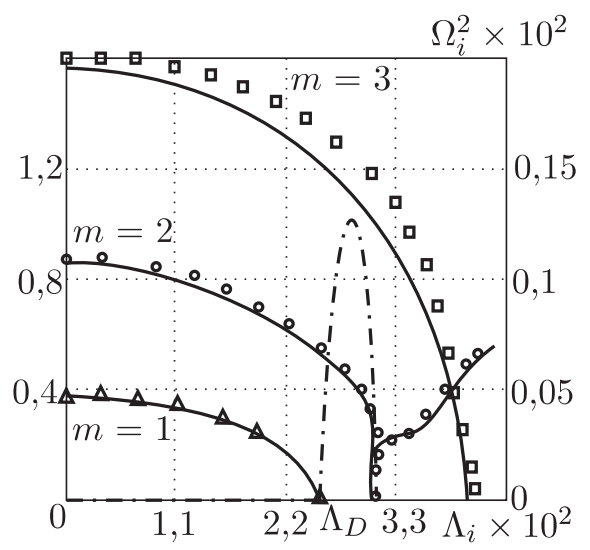

a

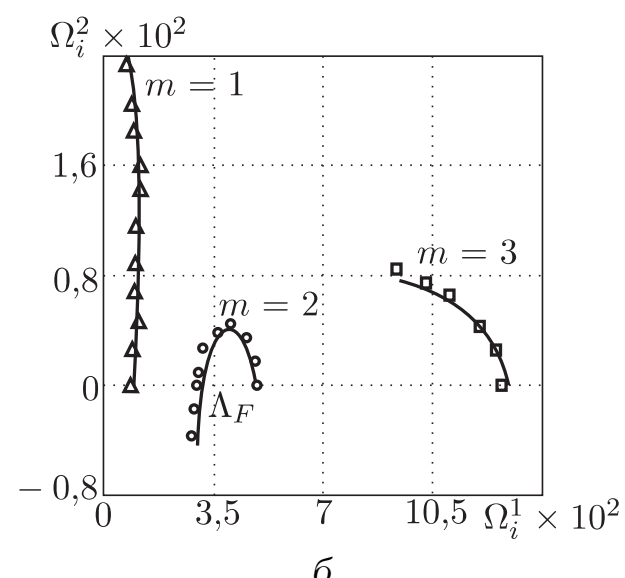

б

Рис. 1. Зависимость действительных $\Omega_{i}^{1}$ и мнимых $\Omega_{i}^{2}$ частей безразмерных собственных значений (a) и диаграммы изменений безразмерных комплексных собственных значений $\Omega_{i}(б)$ от безразмерной скорости $\Lambda_{i}$ при внутреннем течении жёстко закреплённых (а) и консольно закреплённых (б) коаксиальных оболочек: линии - результаты расчёта; маркеры данные из [1] (a), данные из [2] (б) 
В табл. 1 и 2 приведены безразмерные критические скорости дивергентной и флаттерной потери устойчивости $\Lambda_{o}$ для коаксиальных оболочек, взаимодействующих с кольцевым $\left(\Lambda_{o} \neq 0, \Lambda_{i}=0\right)$ и внутренним $\left(\Lambda_{i} \neq 0, \Lambda_{o}=0\right)$ потоками жидкости, полученных для различных вариантов граничных условий и величины кольцевого зазора $k$. Скорости, соответствующие потере устойчивости в виде флаттера по одной форме колебаний, отмечены индексом «*».

Из представленных данных следует, что для любой комбинации граничных условий с уменьшением величины кольцевого зазора снижается также граница потери устойчивости. Причем в случае кольцевого потока жидкости неустойчивость проявляется на более низких скоростях, чем в случае внутреннего течения жидкости. В целом такое поведение обусловлено совокупностью таких факторов, как уменьшение отношения $h / a$, сужение кольцевого зазора, взаимодействие кольцевого потока одновременно с двумя упругими оболочками, разной величиной влияния присоединенных масс жидкости на снижение жёсткости оболочек.

В случае одинаковых граничных условий, задаваемых на торцах обеих оболочек, скорости потери устойчивости для консольно закреплённых оболочек при внутреннем и кольцевом потоке жидкости являются величинами одного порядка. Для других вариантов граничных условий скорости потери устойчивости внутреннего потока существенно превышают скорости в кольцевом потоке. При консольном закреплении свободный край оболочек обеспечивает такую деформированную форму поверхности, которая способствует появлению гидродинамического демпфирования, оказывающего стабилизирующее влияние.

Результаты, представленные в табл. 2, уточняют установленный в [1] факт, что в случае внутреннего течения жидкости упругость внешней оболочки не оказывает никакого влияния на критические скорости потери устойчивости. Это заключение остается верным, если не рассматривать консольно закреплённые оболочки, для которых критические скорости внутреннего потока жидкости зависят не только от упругости внешней оболочки, но и от граничных условий на ее торцах.

В случае кольцевого потока жидкости для различных комбинаций граничных условий изменение в характере динамического поведения коаксиальных оболочек также определяется консольным закреплением. Если консольное закрепление имеет наружная оболочка, то в случае свободно опёртой (SS$\mathrm{CF})$ или жёстко закреплённой (CC-CF) внутренней оболочки это приводит к незначительному повышению критических скоростей по сравнению с одинаковыми граничными условиями, а для противоположных вариантов (CF-SS и $\mathrm{CF}-\mathrm{CC})$, наоборот, к снижению.

Если для одинаковых граничных условий вид потери устойчивости однозначно определяется их вариантом - дивергенция или флаттер, то в случае смешанных граничных условий имеет место более сложная картина. При задании на торцах оболочек граничных условий в виде жёсткого закрепления или свободного опирания в любой комбинации потеря устойчивости осуществляется в виде дивергенции. Консольное закрепление хотя бы одной из оболочек может менять дивергентный вид потери устойчивости на флаттерный. Для внутреннего течения жидкости этот вид неустойчивости реализуется только в случае консольного закрепления внутренней оболочки, тогда как 
Таблица 1

Безразмерные критические скорости кольцевого течения жидкости $\Lambda_{o} \cdot 10^{3}$ для коаксиальных оболочек с различными вариантами граничных условий

\begin{tabular}{|c|c|c|c|c|c|c|c|c|c|c|}
\hline \multirow{4}{*}{$\Gamma У_{i}$} & \multirow{4}{*}{$j$} & \multicolumn{9}{|c|}{ Граничные условия, заданные на торцах внешней оболочки ГУ } \\
\hline & & \multicolumn{3}{|c|}{$\mathrm{CC}$} & \multicolumn{3}{|c|}{$\mathrm{CF}$} & \multicolumn{3}{|c|}{ SS } \\
\hline & & \multicolumn{9}{|c|}{$k$} \\
\hline & & $1 / 2$ & $1 / 10$ & $1 / 20$ & $1 / 2$ & $1 / 10$ & $1 / 20$ & $1 / 2$ & $1 / 10$ & $1 / 20$ \\
\hline \multirow{5}{*}{$\mathrm{CC}$} & 1 & 35,941 & 18,211 & 13,079 & $63,607^{*}$ & $39,865^{*}$ & $28,626^{*}$ & 27,741 & 13,980 & 10,040 \\
\hline & 2 & 20,264 & 10,642 & 7,689 & $26,653^{*}$ & $21,832^{*}$ & $23,589^{*}$ & 14,267 & 7,425 & 5,354 \\
\hline & 3 & 18,070 & 8,406 & 5,986 & $28,677^{*}$ & $15,911^{*}$ & $11,620^{*}$ & 13,581 & 6,933 & 4,955 \\
\hline & 4 & 19,580 & 9,543 & 6,773 & $22,750^{*}$ & $18,646^{*}$ & $15,826^{*}$ & 17,016 & 8,850 & 6,294 \\
\hline & 5 & 21,233 & 11,130 & 7,864 & $26,015^{*}$ & $21,124^{*}$ & $19,901^{*}$ & 19,917 & 10,845 & 7,666 \\
\hline \multirow{5}{*}{$\mathrm{CF}$} & 1 & $63,315^{*}$ & $39,465^{*}$ & $28,625^{*}$ & $64,042^{*}$ & $40,466^{*}$ & $29,372^{*}$ & $57,500^{*}$ & $36,266^{*}$ & $29,503^{*}$ \\
\hline & 2 & $31,412^{*}$ & $29,161^{*}$ & $15,170^{*}$ & $39,095^{*}$ & $27,079^{*}$ & $25,413^{*}$ & $24,953^{*}$ & $19,851^{*}$ & $14,676^{*}$ \\
\hline & 3 & $12,891^{*}$ & 8,361 & 6,089 & $32,255^{*}$ & $23,730^{*}$ & $15,788^{*}$ & 13,615 & $15,399^{*}$ & $12,988^{*}$ \\
\hline & 4 & $16,329^{*}$ & $8,292^{*}$ & $8,026^{*}$ & $35,071^{*}$ & $20,210^{*}$ & $17,001^{*}$ & 17,024 & 9,167 & $10,474^{*}$ \\
\hline & 5 & 21,234 & $11,635^{*}$ & $9,478^{*}$ & $40,105^{*}$ & $23,153^{*}$ & $20,863^{*}$ & 19,921 & $9,873^{*}$ & $11,625^{*}$ \\
\hline \multirow{5}{*}{ SS } & 1 & 24,980 & 13,670 & 9,927 & $57,319^{*}$ & $40,493^{*}$ & $29,473^{*}$ & 21,667 & 11,512 & 8,333 \\
\hline & 2 & 14,044 & 7,303 & 5,302 & $23,943^{*}$ & $20,052^{*}$ & $14,750^{*}$ & 11,678 & 5,888 & 4,254 \\
\hline & 3 & 18,079 & 7,462 & 5,139 & $18,327^{*}$ & $17,541^{*}$ & $4,868^{*}$ & 13,490 & 6,171 & 4,299 \\
\hline & 4 & 19,585 & 9,163 & 6,404 & $14,649^{*}$ & $8,273^{*}$ & $5,346^{*}$ & 16,975 & 8,302 & 5,820 \\
\hline & 5 & 21,247 & 11,023 & 7,731 & $19,896^{*}$ & $10,456^{*}$ & $7,256^{*}$ & 19,903 & 10,525 & 7,383 \\
\hline
\end{tabular}


Таблица 2

Безразмерные критические скорости внутреннего течения жидкости $\Lambda_{i} \cdot 10^{3}$ для коаксиальных оболочек с различными вариантами граничных условий

\begin{tabular}{|c|c|c|c|c|c|c|c|c|c|c|}
\hline \multirow{4}{*}{$\Gamma \mathrm{Y}_{i}$} & \multirow{4}{*}{$j$} & \multicolumn{9}{|c|}{ Граничные условия, заданные на торцах внешней оболочки ГУ } \\
\hline & & \multicolumn{3}{|c|}{$\mathrm{CC}$} & \multicolumn{3}{|c|}{$\mathrm{CF}$} & \multicolumn{3}{|c|}{ SS } \\
\hline & & \multicolumn{9}{|c|}{$k$} \\
\hline & & $1 / 2$ & $1 / 10$ & $1 / 20$ & $1 / 2$ & $1 / 10$ & $1 / 20$ & $1 / 2$ & $1 / 10$ & $1 / 20$ \\
\hline \multirow{5}{*}{$\mathrm{CC}$} & 1 & 73,735 & 71,098 & 67,223 & 73,801 & 71,162 & 68,274 & 73,801 & 71,162 & 68,274 \\
\hline & 2 & 30,417 & 33,241 & 33,737 & 30,417 & 33,242 & 33,770 & 30,417 & 33,242 & 33,770 \\
\hline & 3 & 27,028 & 22,371 & 22,023 & 27,028 & 22,391 & 22,047 & 27,028 & 22,370 & 22,047 \\
\hline & 4 & 28,738 & 22,315 & 21,714 & 28,738 & 22,336 & 21,741 & 28,738 & 22,316 & 21,741 \\
\hline & 5 & 32,067 & 23,758 & 22,775 & 32,067 & 23,780 & 22,808 & 32,067 & 23,758 & 22,808 \\
\hline \multirow{5}{*}{$\mathrm{CF}$} & 1 & $51,503^{*}$ & $64,407^{*}$ & $47,098^{*}$ & $66,768^{*}$ & $70,451^{*}$ & $68,274^{*}$ & $77,008^{*}$ & $61,940^{*}$ & $64,883^{*}$ \\
\hline & 2 & $36,277^{*}$ & $27,874^{*}$ & $26,441^{*}$ & $26,900^{*}$ & $29,295^{*}$ & $29,742^{*}$ & $37,525^{*}$ & $19,760^{*}$ & $23,785^{*}$ \\
\hline & 3 & $31,557^{*}$ & $25,454^{*}$ & $11,806^{*}$ & $31,091^{*}$ & $18,552^{*}$ & $17,697^{*}$ & $30,853^{*}$ & $17,651^{*}$ & $18,074^{*}$ \\
\hline & 4 & $32,738^{*}$ & $18,446^{*}$ & $8,404^{*}$ & $34,008^{*}$ & $22,131^{*}$ & $19,527^{*}$ & $21,912^{*}$ & $24,510^{*}$ & $8,912^{*}$ \\
\hline & 5 & $36,279^{*}$ & $16,554^{*}$ & $9,383^{*}$ & $38,535^{*}$ & $26,167^{*}$ & $21,503^{*}$ & $21,529^{*}$ & $26,763^{*}$ & $19,248^{*}$ \\
\hline \multirow{5}{*}{ SS } & 1 & 42,212 & 47,977 & 48,817 & 42,212 & 47,977 & 48,817 & 42,212 & 47,979 & 48,817 \\
\hline & 2 & 18,036 & 18,304 & 18,580 & 18,036 & 18,304 & 18,597 & 18,036 & 18,304 & 18,597 \\
\hline & 3 & 22,828 & 17,314 & 16,194 & 22,828 & 17,314 & 16,212 & 22,828 & 17,314 & 16,212 \\
\hline & 4 & 27,520 & 20,012 & 18,930 & 27,520 & 20,012 & 18,953 & 27,520 & 20,012 & 18,953 \\
\hline & 5 & 31,444 & 22,767 & 21,598 & 31,444 & 22,767 & 21,629 & 31,444 & 22,767 & 21,629 \\
\hline
\end{tabular}


для кольцевого течения он определяется не только граничными условиями на внешней оболочке, но и величиной кольцевого зазора.

Для демонстрации особенностей динамического поведения коаксиальных оболочек с комбинированными граничными условиями на рис. 2 показано изменение действительных и мнимых частей безразмерных собственных значений $\Omega_{o}$ от скорости кольцевого потока $\Lambda_{o}$ для варианта граничных условий $\mathrm{CC}-\mathrm{CF}(k=1 / 20, j=5)$. Как и в случае представленного выше примера (рис. 2, a), с увеличением скорости течения жидкости действительные части мод достигают нулевого значения. При этом потеря устойчивости в виде дивергенции не осуществляется, так как обе мнимые составляющие этих мод превышают нулевое значение (рис. 2, б). Детальный анализ эволюции действительных и мнимых частей собственных значений позволяет установить, что при совпадении действительных частей наблюдается существенно немонотонное на небольшом интервале изменение мнимых частей. Продемонстрированные закономерности поведения мнимых частей собственных значений определяют необходимость крайне аккуратных вычислений, так как игнорирование этих особенностей может привести к некорректному определению режимов неустойчивости.

Известно [2], что совместное течение внутреннего и кольцевого потоков в случае коаксиальных оболочек, жёстко закреплённых с двух торцов (СС$\mathrm{CC})$, является аддитивным и приводит к еще большему снижению критических скоростей, а для консольно закреплённых оболочек (CF-CF) оказывает стабилизирующее воздействие. Иллюстрацией этому заключению являются результаты, представленные в табл. 3. Здесь приведены безразмерные критические скорости дивергентной и флаттерной потери устойчивости $\Lambda_{o}$ для коаксиальных оболочек, взаимодействующих с кольцевым и внутренним потоками жидкости, текущими с одинаковой скоростью $\left(\Lambda_{i}=\Lambda_{o} \neq 0\right)$.

Из представленных в табл. 3 результатов следует, что при совместном течении обоих потоков с одинаковой скоростью любая комбинация граничных условий, за исключением CF-CF, обеспечивает снижение устойчивости
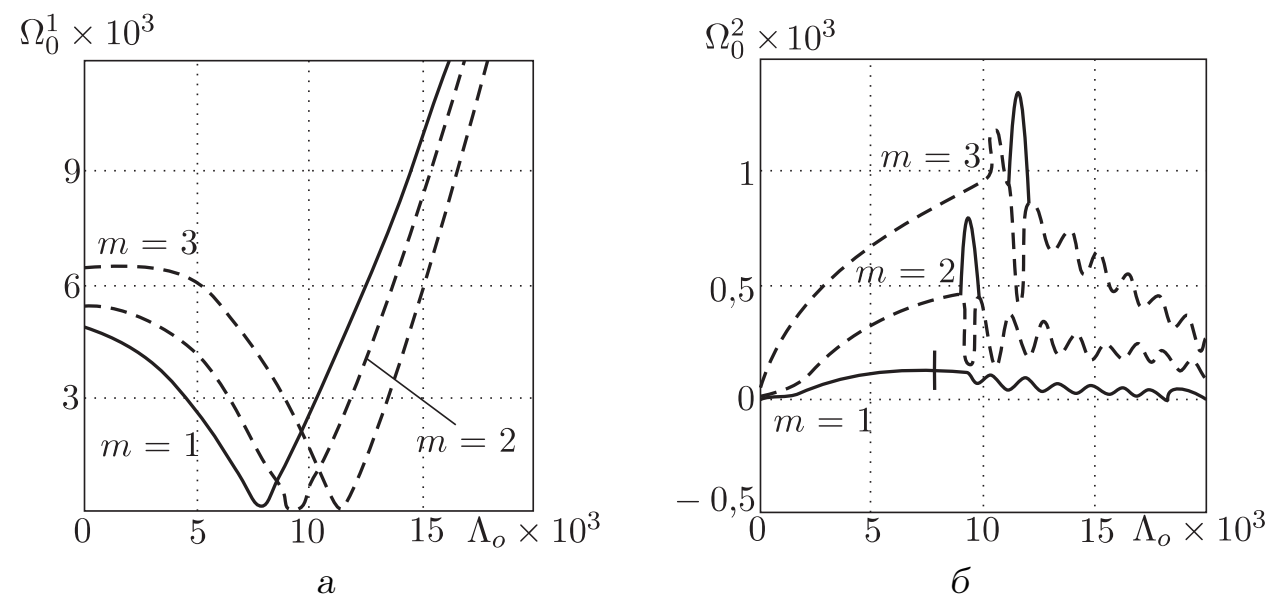

Рис. 2. Зависимость действительных (а) и мнимых (б) частей безразмерных собственных значений $\Omega_{o}$ от безразмерной скорости течения жидкости $\Lambda_{o}$ для коаксиальных оболочек с граничными условиями СC-CF $(k=1 / 20, j=5)$ 
Таблица 3

Безразмерные критические скорости совместного течения внутреннего и кольцевого потоков жидкости $\Lambda \cdot 10^{3}=\Lambda_{i}=\Lambda_{o}$ для коаксиальных оболочек с различными вариантами граничных условий

\begin{tabular}{|c|c|c|c|c|c|c|c|c|c|c|}
\hline \multirow{4}{*}{$\Gamma У_{i}$} & \multirow{4}{*}{$j$} & \multicolumn{9}{|c|}{ Граничные условия, заданные на торцах внешней оболочки ГУ } \\
\hline & & \multicolumn{3}{|c|}{$\mathrm{CC}$} & \multicolumn{3}{|c|}{$\mathrm{CF}$} & \multicolumn{3}{|c|}{ SS } \\
\hline & & \multicolumn{9}{|c|}{$k$} \\
\hline & & $1 / 2$ & $1 / 10$ & $1 / 20$ & $1 / 2$ & $1 / 10$ & $1 / 20$ & $1 / 2$ & $1 / 10$ & $1 / 20$ \\
\hline \multirow{5}{*}{$\mathrm{CC}$} & 1 & 33,720 & 17,960 & 12,998 & $56,758^{*}$ & $37,635^{*}$ & $27,538^{*}$ & 27,159 & 13,915 & 10,017 \\
\hline & 2 & 17,735 & 10,359 & 7,587 & $25,048^{*}$ & $21,236^{*}$ & $20,177^{*}$ & 13,903 & 7,393 & 5,340 \\
\hline & 3 & 16,836 & 8,112 & 5,876 & $25,089^{*}$ & 8,369 & 6,147 & 13,463 & 6,834 & 4,917 \\
\hline & 4 & 18,555 & 9,119 & 6,610 & $21,435^{*}$ & $19,269^{*}$ & $11,882^{*}$ & 16,847 & 8,578 & 6,188 \\
\hline & 5 & 20,826 & 10,554 & 7,635 & $26,191^{*}$ & 10,560 & $14,042^{*}$ & 19,825 & 10,362 & 7,472 \\
\hline \multirow{5}{*}{$\mathrm{CF}$} & 1 & $62,530^{*}$ & $37,616^{*}$ & $27,533^{*}$ & $63,150^{*}$ & $37,770^{*}$ & $27,925^{*}$ & $22,441^{*}$ & $9,990^{*}$ & $5,606^{*}$ \\
\hline & 2 & $25,391^{*}$ & $23,292^{*}$ & $20,154^{*}$ & 63,701 & $38,335^{*}$ & $28,589^{*}$ & $20,701^{*}$ & $9,809^{*}$ & $4,821^{*}$ \\
\hline & 3 & $19,785^{*}$ & $13,379^{*}$ & $6,591^{*}$ & $51,827^{*}$ & 38,849 & 28,962 & 13,524 & $6,720^{*}$ & $3,954^{*}$ \\
\hline & 4 & 29,043 & $19,438^{*}$ & $6,026^{*}$ & $49,786^{*}$ & 39,568 & 29,499 & $16,455^{*}$ & $9,757^{*}$ & $9,017^{*}$ \\
\hline & 5 & 20,834 & $13,715^{*}$ & $9,678^{*}$ & $47,693^{*}$ & $40,270^{*}$ & 30,188 & 19,804 & $12,900^{*}$ & $9,612^{*}$ \\
\hline \multirow{5}{*}{ SS } & 1 & 976 & 13,279 & 9,782 & $42,569^{*}$ & $37,627^{*}$ & $28,124^{*}$ & 20,042 & 11,342 & 8,272 \\
\hline & 2 & 11,280 & 6,871 & 5,139 & 13,033 & $15,125^{*}$ & $14,495^{*}$ & 10,370 & 5,730 & 4,197 \\
\hline & 3 & 14,969 & 7,041 & 4,973 & $14,675^{*}$ & $8,029^{*}$ & $10,119^{*}$ & 13,328 & 5,996 & 4,227 \\
\hline & 4 & 18,109 & 8,655 & 6,201 & $13,883^{*}$ & $9,707^{*}$ & $8,959^{*}$ & 16,792 & 7,974 & 5,688 \\
\hline & 5 & 20,723 & 10,413 & 7,482 & $15,036^{*}$ & $10,881^{*}$ & $10,235^{*}$ & 19,807 & 10,019 & 7,172 \\
\hline
\end{tabular}


системы коаксиальных оболочек по сравнению с вариантами, когда каждый из потоков течет по отдельности. Кроме этого, при таком виде течения потоков не существует комбинации граничных условий, обеспечивающих системе только флаттерный вид потери устойчивости.

Заключение. Для исследования динамического поведения круговых коаксиальных оболочек вращения, взаимодействующих со сжимаемым потоком жидкости, который течет во внутренней оболочке, либо в кольцевом зазоре между оболочками, или в случае совместного течения потоков рассмотрена математическая постановка задачи и конечно-элементный алгоритм ее численной реализации. Полученные результаты сопоставимы с известными численно-аналитическими решениями для оболочек с различными граничными условиями. Представлены результаты численных экспериментов, выполненных для коаксиальных оболочек с различными комбинациями граничных условий при разных значениях кольцевого зазора между оболочками. Установлено, что для рассмотренных конфигураций наибольшее влияние как на критические скорости потери устойчивости, так и на вид потери устойчивости оказывают граничные условия консольного закрепления.

\section{БИБЛИОГРАФИЧЕСКИЙ СПИСОК}

1. Païdoussis M.P., Chan S.P., Misra A.K. Dynamics and stability of coaxial cylindrical shells containing flowing fluid // J. Sound Vib., 1984. Vol. 97, no. 2. Pp. 201-235.

2. Païdoussis M.P., Nguyen V.B., Misra A.K. A theoretical study of the stability of cantilevered coaxial cylindrical shells conveying fluid // J. Fluid Struct., 1991. Vol. 5, no. 2. Pp. 127-164.

3. Païdoussis M.P., Misra A.K., Chan S.P. Dynamics and stability of coaxial cylindrical shells conveying viscous fluid // J. Appl. Mech., 1985. Vol. 52, no. 2. Pp. 389-396.

4. Païdoussis M.P., Misra A.K., Nguyen V. B. Internal- and annular-flow-induced instabilities of a clamped-clamped or cantilevered cylindrical shell in a coaxial conduit: The effects of system parameters // J. Sound Vib., 1992. Vol. 159, no. 2. Pp. 193-205.

5. Nguyen V.B., Païdoussis M.P., Misra A.K. A CFD-based model for the study of the stability of cantilevered coaxial cylindrical shells conveying viscous fluid // J. Sound Vib., 1994. Vol. 176, no. 1. Pp. 105-125.

6. Бочкарёв C. А., Матвеенко В. П. Динамическое поведение упругих коаксиальных цилиндрических оболочек, содержащих движущуюся в них жидкость // ПMМ, 2010. Т. 74, № 4. C. 655-666; англ. пер.: Bochkarev S. A., Matveyenko V.P. The dynamic behaviour of elastic coaxial cylindrical shells conveying fluid // J. Appl. Math. Mech., 2010. Vol. 74, no. 4. Pp. 467-474.

7. Бочкарёв C.А., Матвеенко В.П. Анализ устойчивости нагруженных коаксиальных цилиндрических оболочек с внутренним течением жидкости // Изв. РАН. MTT, 2010. №6. C. 29-45; англ. пер.: Bochkarev S. A., Matveenko V.P. Stability analysis of loaded coaxial cylindrical shells with internal fluid flow // Mech. Solids, 2010. Vol. 45, no. 6. Pp. 789802.

8. El Chebair A., Païdoussis M.P., Misra A.K. Experimental study of annular-flow-induced instabilities of cylindrical shells // J. Fluid Struct., 1989. Vol. 3, no. 4. Pp. 349-364.

9. Nguyen V.B.,Païdoussis M.P., Misra A.K. An experimental study of the stability of cantilevered coaxial cylindrical shells conveying fluid // J. Fluid Struct., 1993. Vol. 7, no. 8. Pp. 913-930.

10. Вольмир А. С. Оболочки в потоке жидкости и газа. Задачи гидроупругости. М.: Наука, 1979. 320 c. [Vol'mir A.S. Shells in Fluid and Gas Flow. Problems of Hydroelasticity. Moscow: Nauka, 1979. 320 pp.] 
11. Бочкарёв C. А., Матвеенко В. П. Численное исследование влияния граничных условий на динамику поведения цилиндрической оболочки с протекающей жидкостью // Изв. PAH. MTT, 2008. № 3. C. 189-199; англ. пер.: Bochkarev S. A., Matveenko V. P. Numerical study of the influence of boundary conditions on the dynamic behavior of a cylindrical shell conveying a fluid // Mech. Solids, 2008. Vol.43, no. 3. Pp. 477-486.

12. Бидерман В.Л. Механика тонкостенных конструкций. М.: Машиностроение, 1977. 488 c. [Biderman V. L. Mechanics of thin-walled structures. Moscow: Mashinostroenie, 1977. 488 pp.]

13. Матвеенко В. П. Об одном алгоритме решения задачи о собственных колебаниях упругих тел методом конечных элементов / В сб.: Краевые задачи теории упругости и вязкоупругости. Свердловск: УНЦ АН СССР, 1980. С. 20-24. [Matveenko V. P. On one algorithm for solving the problem of natural vibrations of elastic bodies by finite element method / In: Boundary value problems of elasticity and viscoelasticity. Sverdlovsk: UNC AN SSSR, 1980. Pp. 20-24].

14. Джсрдж A., Лю Дж. Численное решение больших разреженных систем уравнений. М.: Мир, 1984. 333 с. [Dzhordzh A., Liu Dzh. Numerical Solution of Large Sparse Systems of Equations. Moscow: Mir, 1984. 333 pp.]

Поступила в редакцию 26/III/2012;

в окончательном варианте - 13/VI/2012.

MSC: 74F10

\title{
INVESTIGATION OF BOUNDARY CONDITIONS ON STABILITY OF COAXIAL CYLINDRICAL SHELLS INTERACTING WITH FLOWING FLUID
}

\author{
S. A. Bochkarev, S. V. Lekomtsev \\ Institute of Continuous Media Mechanics, Ural Branch of RAS, \\ 1, Acad. Korolev st., Perm, 614013, Russia. \\ E-mails: bochkarev@icmm.ru, lekomtsev@icmm.ru
}

The work is devoted to investigation of the dynamic behavior of elastic coaxial cylindrical shells interacting with an ideal compressible fluid. The shell behavior is examined in the framework of the classical shell theory, using the variational principle of virtual displacements as a mathematical formulation. The fluid is described in terms of potential theory. With this approach, the stated problem reduces to simultaneous solving of four sets of equations using the finite element method. For shells with different boundary conditions the numerical investigations have been carried out to explore the effects of the annular gap on the boundary of hydroelastic stability.

Key words: coaxial shells, potential fluid, finite element method, stability, divergence, flutter.

Original article submitted 26/III/2012;

revision submitted 13/VI/2012.

Sergey A. Bochkarev (Ph. D. (Phys. \& Math.)), Research Scientist, Div. of complex problems of deformable bodies mechanics. Sergey V. Lekomtsev, Postgraduate student, Div. of complex problems of deformable bodies mechanics. 